\title{
On finitely ambiguous Büchi automata *
}

\author{
Christof Löding and Anton Pirogov ** \\ RWTH Aachen University, Templergraben 55, 52062 Aachen, Germany \\ \{loeding, pirogov\}@cs.rwth-aachen.de
}

\begin{abstract}
Unambiguous Büchi automata, i.e. Büchi automata allowing only one accepting run per word, are a useful restriction of Büchi automata that is well-suited for probabilistic model-checking. In this paper we propose a more permissive variant, namely finitely ambiguous Büchi automata, a generalisation where each word has at most $k$ accepting runs, for some fixed $k$. We adapt existing notions and results concerning finite and bounded ambiguity of finite automata to the setting of $\omega$-languages and present a translation from arbitrary nondeterministic Büchi automata with $n$ states to finitely ambiguous automata with at most $3^{n}$ states and at most $n$ accepting runs per word.
\end{abstract}

Keywords: Büchi automata $\cdot$ infinite words $\cdot$ ambiguity

\section{Introduction}

Nondeterministic Büchi automata (NBA) [6] are finite automata for infinite words that have applications in logical decision procedures, in particular in the field of model checking [3], as they can succinctly represent many interesting properties of non-terminating systems with infinite execution traces. In some contexts, unrestricted nondeterminism is problematic, e.g. in probabilistic model checking reasoning about probabilities becomes very difficult under nondeterminism and therefore other models are necessary.

One solution is determinisation of Büchi automata. As deterministic Büchi automata are strictly weaker than NBA, this requires a quite complex translation to automata with different acceptance conditions and incurs a state blow-up of order $2^{n \log n}$ in the worst-case 1416. But determinisation can be avoided because some restricted forms of nondeterminism are also suitable for probabilistic model checking, e.g. limit-deterministic Büchi automata [8, which can be separated into a subset of states that can never accept, but have nondeterministic transitions, and a deterministic subset that contains all of the accepting states, but cannot reach the nondeterministic states again. Such automata can be used with similar algorithms as deterministic automata and are also suitable for the model checking of Markov decision processes 9 .

\footnotetext{
* The final authenticated publication is available online at https://doi.org/10.1007/978-3-319-98654-8_41

** This work is supported by the German research council (DFG) Research Training Group 2236 UnRAVeL
} 
Another well-studied variant are unambiguous Büchi automata (UBA), i.e. automata admitting at most one accepting run for each word, which are known to be as powerful as unrestricted Büchi automata [2], while they can be exponentially smaller than equivalent deterministic automata 4. On finite words, unambiguous automata form an interesting subclass of nondeterministic automata because they admit a polynomial time inclusion test [15] (while this problem is PSPACE-complete for general nondeterministic automata). This result can be extended to finitely ambiguous automata, which have at most $k$ accepting runs for each input for some fixed number $k$ [15. It is unknown whether the polynomial time inclusion test can be extended to UBA. However, some positive results have been obtained for simpler types of acceptance conditions [10] and a stronger notion of ambiguity [5]. Furthermore, UBA admit a polynomial time algorithm for quantitative probabilistic model checking based on linear equation systems [4].

While standard translations from the temporal logic LTL to NBA yield unambiguous automata, for the transformation of a given NBA into an UBA only non-trivial constructions [1112] roughly of order $n^{n}$ for an $n$ state NBA are known.

In this article, we study finitely ambiguous NBA. To the best of our knowledge, this model has not been considered before. We show that there is a simple construction for transforming any given NBA with $n$ states into a finitely ambiguous NBA with at most $3^{n}$ states. We also present an exponential lower bound of order $2^{n}$ for such a construction, which is easily obtained from a corresponding lower bound for finitely ambiguous automata on finite words [13. Furthermore, we study the possible degrees of ambiguity for NBA. We present a classification of the degree of ambiguity of Büchi automata and the complexity of the corresponding decision problems, based on results for finite words in [17,17. While many results can be generalized from finite to infinite words in a straightforward way, there are different types of infinite degree of ambiguity for NBA. We characterize those in terms of state patterns similar to those that are used over finite words to distinguish between polynomial and exponential growth rates of ambiguity.

This paper is organized as follows. After introducing basic notation in Section 2 we present the classification degrees of ambiguity of nondeterministic Büchi automata and the complexity of the corresponding decision problems in Section 3 . In Section 4 we present the translation from an arbitrary NBA to a finitely ambiguous NBA, and state a lower bound for such a transformation. In Section 5 we conclude. Full versions of proofs sketched in the main text can be found in the appendix.

\section{Preliminaries}

For a finite alphabet $\Sigma, \Sigma^{*}$ denotes the set of all finite and $\Sigma^{\omega}$ the set of all infinite words over $\Sigma$. For $a_{i} \in \Sigma$ and a (finite or infinite) word $w=a_{1} a_{2} \ldots$ let $w(i):=a_{i}$. We denote the prefix of length $i$ with $\operatorname{Pref}_{i}(w):=a_{1} \ldots a_{i}$. The suffix starting at position $i$ is denoted by $\operatorname{Suf}_{i}(w):=a_{i} a_{i+1} \ldots$ Let $\operatorname{Inf}(w)=$ $\{x \mid w(i)=x$ for infinitely many $i\}$ denote the infinity set of a word $w$. 
Let a tuple $\mathcal{A}=\left(Q, \Sigma, \Delta, Q_{0}, F\right)$ denote a finite automaton with some finite alphabet $\Sigma$, finite set of states $Q$, transition relation $\Delta \subseteq Q \times \Sigma \times Q$ and initial and final states $Q_{0}, F \subseteq Q$. Let $|\mathcal{A}|:=|\Delta|$ denote the size of $\mathcal{A}$. We write $\Delta(P, x)$ for $\{q \mid p \in P,(p, x, q) \in \Delta\}, \Delta_{S}(P, x)=\Delta(P, x) \cap S$ and $\Delta_{\bar{S}}(P, x)=\Delta(P, x) \backslash S$ for some $S \subseteq Q$. For convenience, we write $\Delta(p, x)$ when we mean $\Delta(\{p\}, x)$. With $\mathcal{A}[S]$ we denote the modified automaton with $Q_{0}=S, \mathcal{A}[\{s\}]$ can be written as $\mathcal{A}[s]$.

A transition sequence $\pi=\left(q_{1}, a_{1}, r_{1}\right) \ldots\left(q_{n}, a_{n}, r_{n}\right)$ is called a path if $q_{i}$ equals $r_{i-1}$ for all $1<i \leq n$. The source and target of the path are denoted by $\operatorname{src}(\pi)=q_{1}$ and $\operatorname{trg}(\pi)=r_{n}$ while $\operatorname{lbl}(\pi)=a_{1} \ldots a_{n}$ and $\operatorname{st}(\pi)=q_{1} \ldots q_{n} r_{n}$ denote the label and state sequence of $\pi$, respectively. For convenience, let $\pi(i):=$ st $(\pi)(i)$. We also consider infinite paths (with the obvious definition). In general, when speaking of a path or a sequence, we refer to a finite or infinite path or sequence, depending on the context.

The set of all paths from states in $Q$ to states in $R$ labelled with $x$ is denoted by $P(Q, x, R):=\{\pi \mid \operatorname{src}(\pi) \in Q, \operatorname{lbl}(\pi)=x, \operatorname{trg}(\pi) \in R\}$, while $P^{\omega}(Q, x):=$ $\left\{\pi \mid \operatorname{src}(\pi) \in Q, \operatorname{lbl}(\pi)=x, x \in \Sigma^{\omega}\right\}$ denotes all infinite paths with label $x$ starting in a state from $Q$. Paths compose in the expected way. We write $p \stackrel{x}{\rightarrow} q$ if $P(p, x, q) \neq \emptyset$ and $p \rightarrow q$ if $p \stackrel{x}{\rightarrow} q$ for some $x \in \Sigma^{*}$. A strongly connected component (SCC) $C \subseteq Q$ of $\mathcal{A}$ as usual is a maximal (w.r.t. inclusion) subset of states such that if $p, q \in C$ then $p \rightarrow q$ and $q \rightarrow p$.

The language of $\mathcal{A}$ when read as NFA is defined as $L\left(\mathcal{A}_{\mathrm{NFA}}\right):=\left\{x \in \Sigma^{*} \mid\right.$ $\left.P\left(Q_{0}, x, F\right) \neq \emptyset\right\}$. The $\omega$-language of $\mathcal{A}$ when read as nondeterministic Büchi automaton (NBA) is defined as $L\left(\mathcal{A}_{\mathrm{NBA}}\right):=\left\{x \in \Sigma^{\omega} \mid \exists \pi \in P^{\omega}\left(Q_{0}, x\right), \operatorname{lnf}(\operatorname{st}(\pi)) \cap\right.$ $F \neq \emptyset\}$. The set $\operatorname{Runs}(\mathcal{A}, x)$ contains accepting runs of $\mathcal{A}$ on $x$, i.e. all paths from an initial state that are labelled with $x$ and satisfy the corresponding acceptance condition. We say that a set of runs is separated (at time $i$ ) when the prefixes of length $i$ of those runs are pairwise different.

We say $\mathcal{A}$ is trim, if each path from an initial state is a prefix of an accepting run and if $\mathcal{A}$ is an NBA we additionally require that each accepting state is on a cycle. This means for NFA that an accepting state is always reachable and for NBA that a cycle with an accepting state is always reachable and no state is uselessly marked as accepting. In the following, let $\mathcal{A}=\left(Q, \Sigma, \Delta, Q_{0}, F\right)$ be some arbitrary finite automaton if not specified otherwise.

\section{Ambiguity of Büchi automata}

We first give some basic definitions concerning the degree of ambiguity of NFA and NBA. Then we restate some results on the ambiguity of NFA given in [17, and continue with the analysis of degrees of ambiguity for NBA.

By $\aleph_{0}$ we denote the cardinality of the natural numbers and by $2^{\aleph_{0}}$ the cardinality of the real numbers. The degree of ambiguity of automaton $\mathcal{A}$ on a word $x$ is defined as $\operatorname{da}(\mathcal{A}, x):=|\operatorname{Runs}(\mathcal{A}, x)|$ and the degree of ambiguity of an automaton is given by $\operatorname{da}(\mathcal{A}):=\sup _{x}\{\mathrm{da}(\mathcal{A}, x)\}$ over all possible words $x$. Note that the result depends on whether we consider $\mathcal{A}$ to be an NFA or an NBA - in 
the first case we consider finite input words, in the second case infinite words. If $\mathrm{da}(\mathcal{A})<\aleph_{0}, \mathcal{A}$ is finitely ambiguous. We say $\mathcal{A}$ is $k$-ambiguous if $\mathrm{da}(\mathcal{A})=k$ and unambiguous for $k=1$. If $\mathrm{da}(\mathcal{A}) \geq \aleph_{0}, \mathcal{A}$ is infinitely ambiguous.

For infinitely ambiguous $\mathcal{A}_{\text {NFA }}$ let the degree of polynomial ambiguity $\operatorname{dpa}(\mathcal{A})$ be the smallest $k \in \mathbb{N}$ such that for all $w \in \Sigma^{*},|w|=n$, da $(\mathcal{A}, w) \in \mathcal{O}\left(n^{k}\right)$. $\mathcal{A}_{\mathrm{NFA}}$ is polynomially ambiguous if $\operatorname{dpa}(\mathcal{A})<\infty$, otherwise exponentially ambiguous.

So polynomial ambiguity on finite words means that there is no constant upper bound on the number of accepting runs that holds for all words, but there is a polynomial function bounding the number of accepting runs for words with a fixed length. Similarly, exponential ambiguity means that no such polynomial bound exists. As shown in [17, the different types of ambiguity for NFA can be characterized by the following state patterns:

Definition 1 (Infinite ambiguity conditions for NFA).

- $\mathcal{A}$ satisfies IDA (infinite degree of ambiguity) if there are $p, q \in Q, p \neq q, v \in$ $\Sigma^{*}$ and three paths $\pi_{1} \in P(p, v, p), \pi_{2} \in P(p, v, q), \pi_{3} \in P(q, v, q)$. We call a tuple $\left(p, q, v, \pi_{1,2,3}\right)$ an IDA pattern.

- $\mathcal{A}$ satisfies EDA (exponential degree of ambiguity) if there is $p \in Q, v \in \Sigma^{*}$ and two cycles $\pi_{1}, \pi_{2} \in P(p, v, p), \pi_{1} \neq \pi_{2}$. We call a tuple $\left(p, v, \pi_{1,2}\right)$ an EDA pattern.

The corresponding paths $\pi_{i}$ may be omitted when not required.

Theorem 1 ([17]).

1. If $\mathcal{A}$ satisfies EDA, then $\mathcal{A}$ satisfies IDA.

2. $\mathcal{A}$ satisfies $\mathrm{EDA} \Leftrightarrow \mathcal{A}_{\mathrm{NFA}}$ is exponentially ambiguous.

3. $\mathcal{A}$ satisfies $\neg \mathrm{EDA}$ and IDA $\Leftrightarrow \mathcal{A}_{\mathrm{NFA}}$ is polynomially ambiguous.

4. $\mathcal{A}$ satisfies $\neg \mathrm{IDA} \Leftrightarrow \mathcal{A}_{\mathrm{NFA}}$ is finitely ambiguous.

A novel aspect when measuring ambiguity of Büchi automata is that there are multiple degrees of infinite ambiguity when considering infinite words, as a single infinite word can have infinitely many different accepting runs, which is not possible with finite words. In fact, for an infinite word the number of accepting runs can even be uncountable. We will see that for each infinite word the cardinality of the set of different accepting runs is either finite, equal to $\aleph_{0}$ or equal to $2^{\aleph_{0}}$.

Formally, if there exists $w \in \Sigma^{\omega}$ with $\operatorname{da}(\mathcal{A}, w)=2^{\aleph_{0}}$, i.e. some word $w$ has uncountably many accepting runs, we say that $\mathcal{A}$ is uncountably ambiguous and we write $\operatorname{da}(\mathcal{A})=2^{\aleph_{0}}$. If $\mathcal{A}$ is not uncountably ambiguous, but there exists some word $w$ with $\operatorname{da}(\mathcal{A}, w)=\aleph_{0}$, i.e. $w$ has a countably infinite number of accepting runs, $\mathcal{A}_{\mathrm{NBA}}$ is called strict-countably ambiguous. Later we will show that these ambiguity cases can be characterized using the following refinements of the state patterns in Definition 1

\section{Definition 2 (Additional ambiguity conditions for NBA).}

- $\mathcal{A}$ satisfies IDA $F$ if it has an IDA pattern $\left(p, q, v, \pi_{1,2,3}\right)$ such that $q \in F$.

- $\mathcal{A}$ satisfies $\mathrm{EDA}_{F}$ if it has an EDA pattern $\left(p, v, \pi_{1,2}\right)$ such that $p \in F$. 
(a)

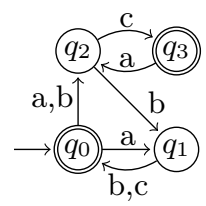

(b)

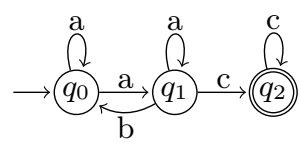

(c)

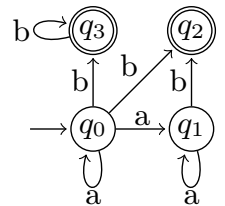

(d)

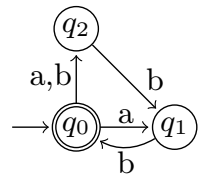

Fig. 1. (a) The word $(a b)^{\omega}$ is accepted unambiguously, while $a b^{\omega}$ has two accepting runs due to the choice of the first transition. The word $(a c)^{\omega}$ has strict-countable ambiguity as $q_{0} \stackrel{a c}{\rightarrow} q_{0}, q_{0} \stackrel{a c}{\rightarrow} q_{3}$ and $q_{3} \stackrel{a c}{\rightarrow} q_{3}$, which is an IDA $_{F}$ pattern. The word $(a c a b b)^{\omega}$ has uncountably many accepting runs due to two paths $q_{0} \stackrel{a c a b b}{\rightarrow} q_{0}$, implying EDA $_{F}$ and therefore the automaton is uncountably ambiguous. (b) This automaton has an IDA pattern $\left(q_{0}, q_{1}, a\right)$ and an EDA pattern $\left(q_{0}, a a b\right)$, but no IDA $_{F}$ nor EDA $F$. The word $a^{*} a c^{\omega}$ is polynomially ambiguous and $(a a b)^{*} a c^{\omega}$ is exponentially ambiguous, as the corresponding pattern can be traversed in different ways a finite number of times, before reading the first $c$. (c) Counterexample of Lemma 1 for non-trim automata: $L\left(\mathcal{A}_{\mathrm{NFA}}\right)=a^{*} b^{+}$and $\mathcal{A}_{\mathrm{NFA}}$ is not finitely ambiguous, because each word $a^{n} b$ has for $0<i \leq n$ the accepting runs $q_{0}^{i} q_{1}^{n-i} q_{2}$, while $L\left(\mathcal{A}_{\mathrm{NBA}}\right)=a^{*} b^{\omega}$ is unambiguous, as the only accepting path must have a state sequence of the form $q_{0}^{*} q_{3}^{\omega}$. (d) As NFA this automaton is unambiguous, while as NBA there are two accepting runs on $a b^{\omega}$.

If the ambiguity of the NBA is not finite, but there are also no infinite words with at least $\aleph_{0}$ accepting runs, we call the NBA limit-countably ambiguous. In this case we can adapt the notions of polynomial and exponential ambiguity. These cases can not be defined in exactly the same way as for NFA because we only consider words that are infinite. But we can still preserve the spirit of the definitions of polynomial and exponential ambiguity by defining them as bounds on the maximal growth of ambiguity with increasing prefix length of words, instead of whole words.

So formally, if $\mathcal{A}_{\text {NBA }}$ is not finitely ambiguous and not at least strict-countably ambiguous, it is limit-countably ambiguous. More specifically, if the function $f$ is an upper bound such that for all $w \in L\left(\mathcal{A}_{\mathrm{NBA}}\right)$ and $i \in \mathbb{N}$ we have $\mid\{\pi \in$ $\left.P\left(Q_{0}, \operatorname{Pref}_{i}(w), Q\right) \mid \operatorname{Runs}\left(\mathcal{A}[\operatorname{trg}(\pi)]_{\mathrm{NBA}}, \operatorname{Suf}_{i+1}(w)\right) \neq \emptyset\right\} \mid \leq f(i)$, we say that $\mathcal{A}_{\mathrm{NBA}}$ it is polynomially ambiguous if one can choose $f(i):=c \cdot i^{d}$ with constants $c$ and $d$, and exponentially ambiguous otherwise.

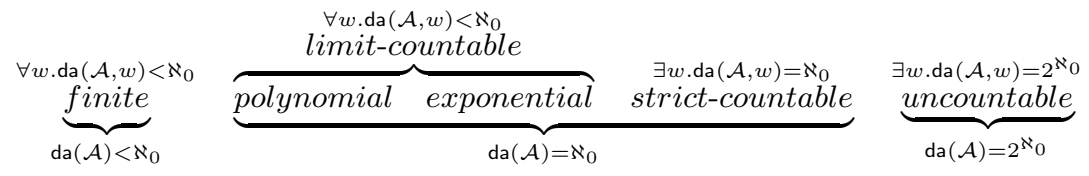

Fig. 2. Illustration of the ambiguity hierarchy for NBA. The five classes (without "limit-countable") are pairwise disjoint. The depicted order reflects the meaning of e.g. "at most polynomial amb.", which includes finite and polynomial ambiguity, or "at least strict-countable amb.", which includes strict-countable and uncountable ambiguity. 
Consider Figure 1 $(a, b)$ to see examples of different ambiguity types on infinite words. In the following we will justify the ambiguity claims in the examples, by relating the patterns from Definition 2 to the various cases that emerge for NBA. The resulting hierarchy is illustrated in Figure 2 and summarized in Theorem 2. We start by establishing that the notion of finite ambiguity of an automaton is closely related for NFA and NBA under the condition that the considered automaton must be trim as NBA.

Lemma 1. Let $\mathcal{A}$ be trim as NBA. Then $\mathcal{A}_{\mathrm{NFA}}$ is finitely ambiguous if and only if $\mathcal{A}_{\mathrm{NBA}}$ is finitely ambiguous.

Proof. For the first claim, assume $\mathcal{A}$ is not a finitely ambiguous NBA. Then for all $k \in \mathbb{N}$ there is a word with at least $k$ different runs. Pick a word $w \in \Sigma^{\omega}$ with at least $k|Q|$ different runs and a time where all these runs are separated. Then there are at least $k$ runs that are in the same state $p \in Q$. We can extend these prefixes by a path from $p$ to some $q \in F$, obtaining a word that is accepted by at least $k$ runs of the corresponding NFA.

The second claim is similar, with the difference that $k$ different finite runs on some finite word that end in the same state are extended to accepting infinite runs (this requires that $\mathcal{A}$ is trim).

See Figure 1 (c) for a non-trim counterexample and also notice that in (d) the automaton has a different finite ambiguity as NFA than as NBA. Hence, in general $\mathrm{da}\left(\mathcal{A}_{\mathrm{NBA}}\right) \neq \mathrm{da}\left(\mathcal{A}_{\mathrm{NFA}}\right)$ and the calculation of the exact degree must also be adapted to the NBA setting. We will sketch a corresponding procedure later in the context of Theorem 4

We now state some technical lemmas for establishing the connection between the state patterns and the degrees of ambiguity summarized in Theorem 2 further below.

Lemma 2. If $\mathcal{A}$ satisfies $\mathrm{EDA}_{F}$, then $\mathcal{A}$ satisfies IDA $_{F}$.

Proof (sketch). Similar argument as for the implication EDA $\Rightarrow$ IDA in [17].

Lemma 3. If $\mathcal{A}$ satisfies $\neg \mathrm{EDA}_{F}$, then for all $q \in F, w \in \Sigma^{\omega}$ the number of infinite paths of $\mathcal{A}[q]$ visiting $q$ infinitely often is at most $|Q|$.

Proof (sketch). Shown by simple construction of an $\mathrm{EDA}_{F}$ pattern in case of more than $|Q|$ such paths.

Now we can relate the extended patterns to the new infinite ambiguity cases.

Lemma 4. $\mathcal{A}$ satisfies $\mathrm{EDA}_{F} \Leftrightarrow \mathcal{A}_{\mathrm{NBA}}$ is uncountably ambiguous. Furthermore, if $\mathcal{A}$ does not satisfy $\mathrm{EDA}_{F}$, then $\mathcal{A}_{\mathrm{NBA}}$ is at most strict-countably ambiguous.

Proof. For one direction, let $\left(p, v, \pi_{1,2}\right)$ be an EDA pattern satisfying EDA . $_{\text {. }}$. Pick some $u \in \Sigma^{*}, \pi_{0} \in P\left(Q_{0}, u, p\right)$. Clearly, $u v^{\omega} \in L\left(\mathcal{A}_{\mathrm{NBA}}\right)$. Observe that for $v^{\omega}=v_{0} v_{1} \ldots$ each $v_{i}$ can be consumed by taking either $\pi_{1}$ or $\pi_{2}$. Hence, the number of runs on $u v^{\omega}$ is uncountable, i.e., $\operatorname{da}\left(\mathcal{A}_{\mathrm{NBA}}\right)=\operatorname{da}\left(\mathcal{A}_{\mathrm{NBA}}, u v^{\omega}\right)=2^{\aleph_{0}}$. 
For the other direction, let $w \in L\left(\mathcal{A}_{\mathrm{NBA}}\right)$ and assume $\mathrm{EDA}_{F}$ does not hold. For each $i \in \mathbb{N}$, let $x=\operatorname{Pref}_{i}(w)$ and notice that the number of different paths $P_{x}=P\left(Q_{0}, x, q\right)$ must be finite for each $q \in F$, as $x$ is finite. By Lemma 3 for each such path there are at most $|Q|$ continuations to infinite runs that visit $q$ infinitely often. It follows that $\mathcal{A}_{\mathrm{NBA}}$ is at most strict-countably ambiguous.

Lemma 5. $\mathcal{A}$ satisfies $I D A_{F} \Leftrightarrow \mathcal{A}_{\mathrm{NBA}}$ is at least strict-countably ambiguous.

Proof (sketch). For one direction, let $\left(p, q, v, \pi_{1,2,3}\right)$ be an IDA pattern satisfying IDA $_{F}$. Let $u \in \Sigma^{*}, \pi_{0} \in P\left(Q_{0}, u, p\right)$. Clearly, $u v^{\omega} \in L\left(\mathcal{A}_{\mathrm{NBA}}\right)$. Observe that for each $i \in \mathbb{N}, \pi_{1}$ can be taken $i$ times before using $\pi_{2}$ and then taking path $\pi_{3}$ forever. Hence, $\operatorname{da}\left(\mathcal{A}_{\mathrm{NBA}}\right) \geq \mathrm{da}\left(\mathcal{A}_{\mathrm{NBA}}, u v^{\omega}\right) \geq \aleph_{0}$.

For the other direction, let $w \in L\left(\mathcal{A}_{\mathrm{NBA}}\right)$ and assume IDA $F$ does not hold. We show that the number of runs on $w$ is finite, and thus the degree of ambiguity of $\mathcal{A}_{\mathrm{NBA}}$ is at most limit-countable. Since IDA $F$ does not hold, EDA $F$ does not hold by Lemma 2 . Then by Lemma 3 a run can separate into at most $|Q|$ different accepting runs that visit $q \in F$ infinitely often, after seeing $q$ the first time. Assume that there is some state $q \in F$ such that there are infinitely many accepting runs that visit $q$ infinitely often. Then there must be such runs for which the first visit to $q$ happens arbitrarily late. From that observation one can construct an $\mathrm{IDA}_{F}$ pattern, a contradiction. But then the number of accepting runs on $w$ must be finite.

The relationship of the different state patterns in $\mathcal{A}$ and the corresponding ambiguity classes of the Büchi automaton are summed up in the following theorem:

Theorem 2. Let $\mathcal{A}$ be a trim NBA.

1. $\mathcal{A}$ satisfies $\mathrm{EDA}_{F} \Leftrightarrow \mathcal{A}_{\mathrm{NBA}}$ is uncountably ambiguous.

2. $\mathcal{A}$ satisfies $\neg \mathrm{EDA}_{F}$ and $\mathrm{IDA}_{F} \Leftrightarrow \mathcal{A}_{\mathrm{NBA}}$ is strict-countably ambiguous.

3. $\mathcal{A}$ satisfies $\neg \mathrm{IDA}_{F}$ and $\mathrm{EDA} \Leftrightarrow \mathcal{A}_{\mathrm{NBA}}$ is exponentially ambiguous.

4. $\mathcal{A}$ satisfies $\neg \mathrm{IDA}_{F}, \neg \mathrm{EDA}$ and IDA $\Leftrightarrow \mathcal{A}_{\mathrm{NBA}}$ is polynomially ambiguous.

5. $\mathcal{A}$ satisfies $\neg \mathrm{IDA}_{F}$ and IDA $\Leftrightarrow \mathcal{A}_{\mathrm{NBA}}$ is limit-countably ambiguous.

6. $\mathcal{A}$ satisfies $\neg \mathrm{IDA} \Leftrightarrow \mathcal{A}_{\mathrm{NBA}}$ is finitely ambiguous.

Proof.

$(1+2)$ : By Lemma 4 and Lemmas 4 and 5 , respectively.

$(3+4)$ : We show both directions by establishing that the difference in growth of ambiguity of $\mathcal{A}_{\text {NFA }}$ in the length of finite words and the growth of ambiguity of $\mathcal{A}_{\mathrm{NBA}}$ in the length of finite prefixes is bounded by constants.

$(\Rightarrow): \neg \mathrm{IDA}_{F}$ implies $\neg \mathrm{EDA}_{F}$ by Lemma 2 and by Lemma $5 \mathcal{A}_{\mathrm{NBA}}$ is not strict-countably ambiguous, hence no word has infinitely many runs. But by Theorem 17 17 there is a family of finite words with increasing length that w.l.o.g. terminate in the same $q \in F$ and witness the polynomial (exponential) ambiguity of $\mathcal{A}_{\text {NFA }}$. As $\mathcal{A}$ is trim, for each such word $u$ with $k$ accepting runs, those runs can be extended by the same loop from $q$ to $q$ labelled with 
some $v$, hence $u v^{\omega}$ has at least $k$ (and by Lemma 3 at most $k|Q|$ ) accepting runs in $\mathcal{A}$ as NBA. Hence $\mathcal{A}_{\text {NBA }}$ has asymptotically the same limit-countable ambiguity.

$(\Leftarrow)$ : Pick some $w \in L\left(\mathcal{A}_{\mathrm{NBA}}\right)$ with $\operatorname{da}\left(\mathcal{A}_{\mathrm{NBA}}, w\right)=k$ and pick $i$ such that after reading the prefix of length $i$ all accepting runs have separated. Notice that there must be at least $\left\lfloor\frac{k}{|Q|}\right\rfloor$ different runs that are in the same state $p$. As $\mathcal{A}$ is trim, there is some finite word $x$ with $|x| \leq|Q|$ leading from $p$ to some $q \in F$. Let $a \leq|Q|^{|Q|}$ be the maximum number of different paths of length $|Q|$ in $\mathcal{A}$ that have the same source, target and label and let $\hat{w}=\operatorname{Pref}_{i}(w) x$. Then on any $x$ each run can separate into at most $a$ different runs and therefore we have that $\left\lfloor\frac{k}{|Q|}\right\rfloor \leq \mathrm{da}\left(\mathcal{A}_{\mathrm{NFA}}, \hat{w}\right) \leq a k$. Hence, by picking for each $i$ an infinite word $w$ with the largest number of separated accepting runs after reading $\operatorname{Pref}_{i}(w)$, we can construct a family of finite words with the number of accepting runs growing asymptotically in the same way as the maximum number of separated accepting runs grows on prefixes of infinite words. By Theorem 17 17 this implies that $\mathcal{A}$ must satisfy IDA or EDA, respectively.

(5): By (3), (4) and Theorem 1, as EDA implies IDA.

(6): Shown in Theorem 1 for trim NFA. By Lemma 1 this extends to trim NBA.

The ambiguity class of an NBA from the hierarchy in Theorem 2 can also be determined efficiently:

\section{Theorem 3.}

1. Uncountable ambiguity of an NBA $\mathcal{A}$ can be decided in $\mathcal{O}\left(|\mathcal{A}|^{2}\right)$.

2. The ambiguity class of an NBA $\mathcal{A}$ can be computed in $\mathcal{O}\left(|\mathcal{A}|^{3}\right)$.

3. $\operatorname{dpa}\left(\mathcal{A}_{\mathrm{NBA}}\right)$ can be computed in $\mathcal{O}\left(|\mathcal{A}|^{3}\right)$.

Proof. A straightforward modification of the corresponding algorithms for NFA from [1], which use a depth-first search in products of $\mathcal{A}$ with itself. EDA $F$ and $I_{F A}$ can easily be checked by adding the corresponding restriction to the IDA / EDA pattern, i.e., that a specific state must additionally be accepting. In case of polynomial ambiguity, the algorithm to calculate $\mathrm{dpa}\left(\mathcal{A}_{\mathrm{NBA}}\right)$ can be used without changes after excluding EDA and IDA ${ }_{F}$.

Computing the exact degree of finite ambiguity, similar to the case for NFA [7, is a difficult problem:

Theorem 4. Deciding whether $\operatorname{da}\left(\mathcal{A}_{\mathrm{NBA}}\right)>d$ for a given automaton $\mathcal{A}$ and $d \in \mathbb{N}$ is a PSPACE-complete problem.

Proof (sketch). We adapt the algorithm presented in [7 to show PSPACEcompleteness of this problem from NFA to NBA. The nondeterministic algorithm for NFA guesses a word with at least $d+1$ accepting runs and evaluates the product of the transition matrices for each symbol along the word (which yields the number of different runs), while bounding the growth of the numbers. First we show that we can restrict ourselves to ultimately periodic words of the form $u v^{\omega}$ 
with $u, v \in \Sigma^{*}$ and then we argue that it suffices to guess $u$ and $v$ accordingly. We show completeness by a simple reduction from the NFA to the NBA problem that maps each finite word $w$ accepted by the NFA one-to-one to a word $w \#^{\omega}$ accepted by the NBA with the same number of accepting runs.

\section{Translation from NBA to finitely ambiguous NBA}

We present a construction that converts a given NBA with $n$ states into a finitely ambiguous NBA with degree of ambiguity at most $n$. For explaining the intuition of the construction and for proving its correctness, we first introduce in Section 4.1 the notion of the reduced split tree as defined in [11. This tree, defined for an NBA $\mathcal{A}$ and an infinite word $w$, collects runs of $\mathcal{A}$ on $w$ in a specific way.

The translation of an NBA into a finitely ambiguous NBA is presented in Section 4.2 The construction uses two disjoint sets for tracking runs of the given NBA, and its description does not rely on the notion of reduced split tree. However, for understanding the role of the two subsets in the construction, reduced split trees are a valuable tool.

\subsection{Reduced Split Trees}

An $X$-labelled binary tree is a partial function $T:\{0,1\}^{*} \rightarrow X$ such that the domain $N_{T}$ of $T$ is a non-empty prefix-closed set. The elements of $N_{T}$ are called the nodes of $T$. The root node is $\varepsilon$ (since $N_{T}$ is prefix closed, $\varepsilon \in N_{T}$ ). For a node $u \in\{0,1\}^{*}$, the node $u 0$ is the left child of $u$, and $u 1$ is the right child of $u$. For two nodes $u, v \in\{0,1\}^{*}$ we say that $u$ and $v$ are on the same level if $|u|=|v|$, and we further say that $u$ is to the left of $v$ if $u$ is lexicographically smaller than $v$.

An infinite path through such a tree corresponds to an infinite sequence $\pi \in\{0,1\}^{\omega}$ (the nodes on the path are the finite prefixes of $\pi$ ). We say that $\pi$ is left-recurring if it contains infinitely many 0 (which means that it moves to the left successor infinitely often).

Let $\mathcal{A}=\left(Q, \Sigma, \Delta, Q_{0}, F\right)$ be an NBA and $w \in \Sigma^{\omega}$ be an infinite word. The following definitions are illustrated by simple examples in Figure 3(a)-(c).

The split tree $T_{\mathrm{s}}^{\mathcal{A}, w}$ is a $2^{Q}$-labelled binary tree with node set $N_{T}=\{0,1\}^{*}$ defined as follows: The root is labelled with the set of initial states $T_{\mathrm{s}}^{\mathcal{A}, w}(\varepsilon):=Q_{0}$. If $u \in\{0,1\}^{*}$ with $|u|=i$ is labelled with $T_{\mathrm{s}}^{\mathcal{A}, w}(u)=P \subseteq Q$, then $T_{\mathrm{s}}^{\mathcal{A}, w}(u 0):=$ $\Delta_{F}(P, w(i))$ and $T_{\mathrm{s}}^{\mathcal{A}, w}(u 1):=\Delta_{\bar{F}}(P, w(i))$.

Observe that $T_{\mathrm{s}}^{\mathcal{A}, w}$ is an infinite complete binary tree that encodes all runs of $\mathcal{A}$ on $w$. It groups the runs by their visits to the set $F$ by continuing runs that pass through an accepting state to the left, and the other ones to the right. Note that $T_{\mathrm{s}}^{\mathcal{A}, w}$ can have nodes with label $\emptyset$, and that a state can occur in many labels on each level.

The reduced left-right tree $T_{\mathrm{rs}}^{\mathcal{A}, w}$ is obtained from $T_{\mathrm{s}}^{\mathcal{A}, w}$ by keeping for each state only the leftmost occurrence on each level (i.e., if $u$ is to the left of $v$, and 
(c)

(a)
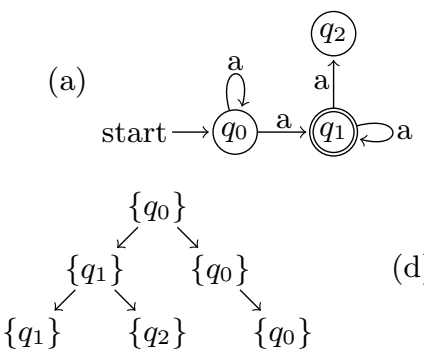

(b)

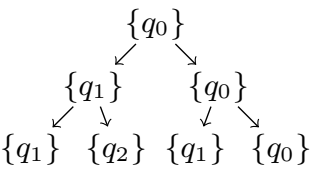

(d) $\left(\emptyset,\left\{q_{1}\right\}\right) \quad\left(\left\{q_{1}\right\},\left\{q_{0}\right\}\right)$

$\left(\emptyset,\left\{q_{1}\right\}\right)\left(\left\{q_{1}\right\},\left\{q_{2}\right\}\right)\left(\left\{q_{1}, q_{2}\right\},\left\{q_{0}\right\}\right)$

Fig. 3. (a) An NBA $\mathcal{A}$ (b) First levels of $T_{\mathrm{s}}^{\mathcal{A}, w}$ for $w=a^{\omega}$ (c) First levels of $T_{\mathrm{rs}}^{\mathcal{A}, w}$ for $w=a^{\omega}$ (d) First two steps of possible transitions of $\mathcal{A}^{\prime}$

$q$ occurs in the labels of $u$ and $v$, then it is removed from the label of $v$ ) and then removing vertices labelled with $\emptyset$. This results in a tree because if the label of a node becomes empty by the above operation, then also the labels of its successors become empty.

The interesting properties of $T_{\mathrm{rs}}^{\mathcal{A}, w}$ are summarized in the following lemma, which is shown in [11.

\section{Lemma 6 ([11]).}

1. The word $w$ is accepted by $\mathcal{A}$ if, and only if, $T_{\mathrm{rs}}^{\mathcal{A}, w}$ contains an infinite leftrecurring path.

2. $T_{\mathrm{rs}}^{\mathcal{A}, w}$ contains at most $|Q|$ infinite paths.

The second claim is obvious because each state of $\mathcal{A}$ is contained in at most one label of each level. The first claim is shown by picking a run of $\mathcal{A}$ on $w$ that always visits the next accepting states as early as possible (details can be found in 11]).

\subsection{Construction of a Finitely Ambiguous NBA}

We construct from $\mathcal{A}$ a new NBA $\mathcal{A}^{\prime}$ whose infinite runs on a word $w$ are in one-to-one correspondence with the infinite paths of $T_{\mathrm{rs}}^{\mathcal{A}, w}$. The run moves into a final state if the corresponding path branches to the left. Then Lemma 6 implies that $\mathcal{A}^{\prime}$ is equivalent to $\mathcal{A}$, and its degree of ambiguity is bounded by $|Q|$.

In order to implement this idea, $\mathcal{A}^{\prime}$ guesses an infinite path through $T_{\mathrm{rs}}^{\mathcal{A}}, w$ by constructing its label sequence. If the current label is $S$ (corresponding to some node $u$ in $T_{\mathrm{rs}}^{\mathcal{A}, w}$ ), then the labels $S_{0}$ and $S_{1}$ of $u 0$ and $u 1$ in $T_{\mathrm{rs}}^{\mathcal{A}, w}$ can be constructed based on the next input letter, the transition relation of $\mathcal{A}$, and the knowledge which states occur in the labels to the left of $u$ in $T_{\mathrm{rs}}^{\mathcal{A}, w}$. This latter information is tracked in a second set $P$. Thus, in $\mathcal{A}^{\prime}$ a state $(P, S)$ for $P, S \subseteq Q$ is reachable by reading the first $i$ letters of an input word $w$ if $S$ is the label of some node $u$ on level $i$ in $T_{\mathrm{rs}}^{\mathcal{A}, w}$, and $P$ is the union of the labels of nodes to the left of $u$ (on the same level). 
Formally, we define the new automaton in the following way. Given NBA $\mathcal{A}$, let $\mathcal{A}^{\prime}=\left(Q^{\prime}, \Sigma, Q_{0}^{\prime}, \Delta^{\prime}, F^{\prime}\right)$ be defined by

- $Q^{\prime}=\left\{(P, S) \in 2^{Q} \times 2^{Q} \mid P \cap S=\emptyset\right.$ and $\left.S \neq \emptyset\right\}$

- $Q_{0}^{\prime}=\left(\emptyset, Q_{0}\right)$

- $\Delta^{\prime}=\Delta_{0}^{\prime} \cup \Delta_{1}^{\prime}$ with

- $\Delta_{0}^{\prime}=\left\{\left((P, S), a,\left(P^{\prime}, S^{\prime}\right)\right) \mid P^{\prime}=\Delta(P, a), S^{\prime}=\Delta_{F}(S, a) \backslash P^{\prime}\right\}$,

- $\Delta_{1}^{\prime}=\left\{\left((P, S), a,\left(P^{\prime}, S^{\prime}\right)\right) \mid P^{\prime}=\Delta(P, a) \cup \Delta_{F}(S, a), S^{\prime}=\Delta_{\bar{F}}(S, a) \backslash P^{\prime}\right\}$

Note that the transitions are only defined if $S^{\prime} \neq \emptyset$ by definition of $Q^{\prime}$.

- $F^{\prime}=\{(P, S) \mid S \subseteq F\}$

Figure 3(d) illustrates the possible transitions of $\mathcal{A}^{\prime}$.

The following lemma is a direct consequence of the definition of $T_{\mathrm{rs}}^{\mathcal{A}, w}$ and the construction of $\mathcal{A}^{\prime}$ (by an induction on $i$ ).

Lemma 7. Let $x$ be the finite prefix of length $i$ of $w$, and assume that $\mathcal{A}^{\prime}$ can reach the state $(P, S)$ by reading $x$ from its initial state. Then there is a node $u$ on level $i$ of $T_{\mathrm{rs}}^{\mathcal{A}, w}$ with label $S$, and $P$ is the union of the labels of the nodes to the left of $u$ on level $i$.

Lemma 7 implies that the infinite runs of $\mathcal{A}^{\prime}$ on $w$ are in one-to-one correspondence with the infinite paths of $T_{\mathrm{rs}}^{\mathcal{A}}, w$ (and therefore $\mathcal{A}^{\prime}$ has degree of ambiguity at most $|Q|$ ). The final states of $\mathcal{A}^{\prime}$ are those in which the second component is a subset of $F$, and therefore correspond to a left successor on the corresponding path in $T_{\mathrm{rs}}^{\mathcal{A}, w}$. Hence, $\mathcal{A}^{\prime}$ has an accepting run of $w$ if, and only if, $T_{\mathrm{rs}}^{\mathcal{A}, w}$ has a left-recurring path. By Lemma 6, this implies that $L(\mathcal{A})=L\left(\mathcal{A}^{\prime}\right)$.

The number of disjoint pairs of subsets of $Q$ is not larger than $3^{|Q|}$. In summary, we obtain the following result.

Theorem 5. Let $\mathcal{A}$ be an NBA with $n$ states. Then there exists an automaton $\mathcal{A}^{\prime}$ with at most $3^{n}$ states accepting the same language such that $\mathrm{da}\left(\mathcal{A}^{\prime}\right) \leq n$.

An exponential lower bound for the construction of finitely ambiguous NBA can be inferred from a corresponding lower bound for NFA.

Theorem 6. For each $n \in \mathbb{N}$ there exists an NBA with $n$ states such that each finitely ambiguous NBA accepting the same language has at least $2^{n}-1$ states.

Proof (sketch). In [13] a family $\left\{\mathcal{A}_{i}\right\}_{i \in \mathbb{N}}$ of NFA is presented such that each $\mathcal{A}_{n}$ has $n$ states, and each at most polynomially ambiguous NFA equivalent to $\mathcal{A}_{n}$ has $2^{n}-1$ states. So the lower bound also holds for finitely ambiguous NFA. This lower bound can be lifted to NBA by considering the languages $L_{n}^{\prime}=\left(L\left(\mathcal{A}_{n}\right) \#\right)^{\omega}$, for which one easily obtains $n$ state NBA from the NFA $\mathcal{A}_{n}$. Furthermore, from a finitely ambiguous NBA for $L_{n}^{\prime}$ one can extract a finitely ambiguous NFA for $L\left(\mathcal{A}_{n}\right)$ with the same number of states.

Our construction is already close to this bound. As it is tracking just two subsets, it is also much simpler than the translations used to obtain unambiguous automata presented in [11]12] that have upper bounds of $4(3 n)^{n}$ and $n(0.76 n)^{n}$ respectively, and are obtained at the cost of much more involved constructions. To the best of our knowledge, it is not known whether there is a stronger lower bound for the construction of unambiguous NBA than the one in Theorem 6 . 


\section{Conclusion}

In this paper we presented a fine classification for the ambiguity of nondeterministic Büchi automata by lifting results known for NFA and extending them to precisely capture the subtle differences in the case of infinite ambiguity. Finally we presented and discussed a translation from NBA to finitely ambiguous NBA. In future work we plan to investigate how this partial disambiguation can be applied in the setting of probabilistic model checking and look for cases in which using finitely ambiguous NBA could have an advantage over full disambiguation or determinisation.

\section{References}

1. Allauzen, C., Mohri, M., Rastogi, A.: General algorithms for testing the ambiguity of finite automata. In: DLT 2008. pp. 108-120. Springer

2. Arnold, A.: Rational $\omega$-languages are non-ambiguous. Theoretical Computer Science 26(1-2), 221-223 (1983)

3. Baier, C., Katoen, J.: Principles of model checking. MIT Press (2008)

4. Baier, C., Kiefer, S., Klein, J., Klüppelholz, S., Müller, D., Worrell, J.: Markov chains and unambiguous Büchi automata. In: CAV 2016. pp. 23-42. Springer

5. Bousquet, N., Löding, C.: Equivalence and inclusion problem for Strongly Unambiguous Büchi Automata. In: LATA 2010. pp. 118-129

6. Büchi, J.R.: On a decision method in restricted second order arithmetic. In: Studies in Logic and the Foundations of Mathematics, vol. 44, pp. 1-11. Elsevier (1966)

7. Chan, T.h., Ibarra, O.H.: On the finite-valuedness problem for sequential machines. Theoretical Computer Science 23(1), 95-101 (1988)

8. Courcoubetis, C., Yannakakis, M.: The complexity of probabilistic verification. Journal of the ACM 42(4), 857-907 (1995)

9. Couvreur, J.M., Saheb, N., Sutre, G.: An optimal automata approach to LTL model checking of probabilistic systems. In: LPAR 2003. pp. 361-375. Springer

10. Isaak, D., Löding, C.: Efficient inclusion testing for simple classes of unambiguous $\omega$-automata. Inf. Process. Lett. 112(14-15), 578-582 (2012)

11. Kähler, D., Wilke, T.: Complementation, disambiguation, and determinization of Büchi automata unified. In: ICALP 2008. pp. 724-735. Springer

12. Karmarkar, H., Joglekar, M., Chakraborty, S.: Improved upper and lower bounds for Büchi disambiguation. In: ATVA 2013. pp. 40-54. Springer

13. Leung, H.: Separating exponentially ambiguous finite automata from polynomially ambiguous finite automata. SIAM Journal on Computing 27(4), 1073-1082 (1998)

14. Safra, S.: On the complexity of omega-automata. In: Foundations of Computer Science, 1988., 29th Annual Symposium on. pp. 319-327. IEEE (1988)

15. Stearns, R.E., Hunt III, H.B.: On the equivalence and containment problems for unambiguous regular expressions, regular grammars and finite automata. SIAM Journal on Computing 14(3), 598-611 (1985)

16. Thomas, W.: Languages, automata, and logic. In: Handbook of formal languages, pp. 389-455. Springer (1997)

17. Weber, A., Seidl, H.: On the degree of ambiguity of finite automata. Theoretical Computer Science 88(2), 325-349 (1991) 


\section{A Full proofs}

In this appendix we provide the full proofs that were omitted in the main paper.

\section{A.1 Proofs for Theorem 2}

Here we provide the full proofs for the technical lemmas that are needed to obtain the ambiguity hierarchy for NBA stated in Theorem 2.

Lemma 8 (Pattern shifting).

1. Iff $\mathcal{A}$ has an IDA pattern $\left(p, q, v, \pi_{1,2,3}\right)$ visiting some $q \in F$ on $\pi_{3}$, then IDA $_{F}$ holds.

2. Iff $\mathcal{A}$ has an EDA pattern $\left(p, v, \pi_{1,2}\right)$ visiting some $q \in F$ on $\pi_{1}$ or $\pi_{2}$, then $\mathrm{EDA}_{F}$ holds.

Proof. $(\Leftarrow)$ holds by definition of IDA $_{F}$ and $\mathrm{EDA}_{F}$.

$(\Rightarrow)$ for $(1)$ : Let $\left(p, q, v, \pi_{1,2,3}\right)$ be an IDA pattern such that $\pi_{3}$ visits $q^{\prime} \in F$. Split $\pi_{3}$ in $q^{\prime} \in F$, i.e. let $\pi_{3}=\pi_{3}^{1} \pi_{3}^{2}$ such that $\operatorname{trg}\left(\pi_{3}^{1}\right)=\operatorname{src}\left(\pi_{3}^{2}\right)=q^{\prime}$ and let $x=l\left(\pi_{3}^{1}\right), y=l\left(\pi_{3}^{2}\right)$. Observe that $v=x y$ and let $\hat{v}=y x$. Now split the path $\pi_{1}$ in the same way, i.e. $\pi_{1}=\pi_{1}^{1} \pi_{1}^{2}$ with $l\left(\pi_{1}^{1}\right)=x, l\left(\pi_{1}^{2}\right)=y$ and let $p^{\prime}$ denote the state $\operatorname{trg}\left(\pi_{1}^{1}\right)$. Observe that we now have three paths $\hat{\pi}_{1}=\pi_{1}^{2} \pi_{1} \pi_{1}^{1} \in$ $P\left(p^{\prime}, \hat{v} \hat{v}, p^{\prime}\right), \hat{\pi}_{2}=\pi_{1}^{2} \pi_{2} \pi_{3}^{1} \in P\left(p^{\prime}, \hat{v} \hat{v}, q^{\prime}\right)$ and $\hat{\pi}_{3}=\pi_{3}^{2} \pi_{3} \pi_{3}^{1} \in P\left(q^{\prime}, \hat{v} \hat{v}, q^{\prime}\right)$, hence $\left(p^{\prime}, q^{\prime}, \hat{v} \hat{v}, \hat{\pi}_{1,2,3}\right)$ is an IDA pattern satisfying IDA $_{F}$.

$(\Rightarrow)$ for $(2)$ : Let $\left(p, v, \pi_{1,2}\right)$ be an EDA pattern that w.l.o.g. visits $p^{\prime} \in F$ on cycle $\pi_{1}$. Now let $\pi_{1}=\pi_{1}^{1} \pi_{1}^{2}$ such that $\operatorname{trg}\left(\pi_{1}^{1}\right)=\operatorname{src}\left(\pi_{1}^{2}\right)=q$ and let $x=l\left(\pi_{1}^{1}\right)$ and $y=l\left(\pi_{1}^{2}\right)$. Observe that $v=x y$ and let $\hat{v}=y x$. Now we can take two different paths $\hat{\pi}_{1}=\pi_{1}^{2} \pi_{1} \pi_{1}^{1}, \hat{\pi}_{2}=\pi_{1}^{2} \pi_{2} \pi_{1}^{1} \in P\left(p^{\prime}, \hat{v} \hat{v}, p^{\prime}\right)$, hence $\left(p^{\prime}, \hat{v} \hat{v}, \hat{\pi}_{1,2}\right)$ is an EDA pattern satisfying $\mathrm{EDA}_{F}$.

Lemma 2. If $\mathcal{A}$ satisfies $\mathrm{EDA}_{F}$, then $\mathcal{A}$ satisfies IDA $_{F}$.

Proof. Similar argument as for EDA $\Rightarrow$ IDA in [17]. Assume that $\left(r, v, \pi_{1,2}\right)$ is an EDA pattern satisfying $\mathrm{EDA}_{F}$, i.e., $r \in F$. As $\pi_{1} \neq \pi_{2}$, let $v=x y$ such that the paths differ after reading $x$ and let $\hat{v}=y x$. Take the first differing state on each of them, call them $p$ and $q$ and split the paths at this position into $\pi_{1}=\pi_{1}^{1} \pi_{1}^{2}, \pi_{2}=\pi_{2}^{1} \pi_{2}^{2}$. Now let $\hat{\pi}_{1}=\pi_{1}^{2} \pi_{1} \pi_{1}^{1}, \hat{\pi}_{2}=\pi_{1}^{2} \pi_{2} \pi_{2}^{1}, \hat{\pi}_{3}=\pi_{2}^{2} \pi_{2} \pi_{2}^{1}$. Notice that each of them visits $r \in F$, hence $\left(p, q, \hat{v} \hat{v}, \hat{\pi}_{1,2,3}\right)$ is an IDA pattern and $r$ is visited on the path $\hat{\pi}_{3}$. This is equivalent to IDA $_{F}$ by Lemma 8

Lemma 3. If $\mathcal{A}$ satisfies $\neg \mathrm{EDA}_{F}$, then for all $q \in F, w \in \Sigma^{\omega}$ the number of infinite paths of $\mathcal{A}[q]$ visiting $q$ infinitely often is at most $|Q|$.

Proof. Let $q \in F$ and $w \in \Sigma^{\omega}$ such that there are $|Q|+1$ different infinite paths of $\mathcal{A}[q]$ visiting $q$ infinitely often. Now pick a time $i$ after which those infinite paths are separated and observe that now there must be two different prefixes $\pi_{1}$ and $\pi_{2}$ of these infinite paths that are in the same state $p \in Q$. Clearly, we can reach $q$ from $p$ again by following one of those two runs to the next visit of $q$ along some path $\pi_{p q}$. But then $\hat{\pi}_{1}:=\pi_{1} \pi_{p q}$ and $\hat{\pi}_{2}:=\pi_{2} \pi_{p q}$ are two different paths from $q$ to $q$ and are labelled by the same prefix $\operatorname{Pref}_{j}(w)$ for some $j>i$ and hence $\left(q, \operatorname{Pref}_{j}(w), \hat{\pi}_{1,2}\right)$ is an EDA pattern satisfying $\operatorname{EDA}_{F}$. 
Lemma 5. $\mathcal{A}$ satisfies $I D A_{F} \Leftrightarrow \mathcal{A}_{\mathrm{NBA}}$ is at least strict-countably ambiguous.

Proof. For one direction, let $\left(p, q, v, \pi_{1,2,3}\right)$ be an IDA pattern satisfying IDA ${ }_{F}$. Let $u \in \Sigma^{*}, \pi_{0} \in P\left(Q_{0}, u, p\right)$. Clearly, $u v^{\omega} \in L\left(\mathcal{A}_{\mathrm{NBA}}\right)$. Observe that for each $i \in \mathbb{N}, \pi_{1}$ can be taken $i$ times before using $\pi_{2}$ and then taking path $\pi_{3}$ forever. Hence, $\operatorname{da}\left(\mathcal{A}_{\mathrm{NBA}}\right)=\operatorname{da}\left(\mathcal{A}_{\mathrm{NBA}}, u v^{\omega}\right) \geq \aleph_{0}$.

For the other direction, let $w \in L\left(\mathcal{A}_{\mathrm{NBA}}\right)$ and assume IDA $_{F}$ does not hold, which implies $\neg \mathrm{EDA}_{F}$ by Lemma 2. Then by Lemma 3 a run can separate into at most $|Q|$ different accepting runs that visit $q \in F$ infinitely often, after seeing $q$ the first time.

For contradiction, assume that there is some state $q \in F$ such that there are infinitely many accepting runs that visit $q$ infinitely often. This requires that the first visit of $q$ by a run can be delayed for an arbitrarily long time. But then there must exist an infinite path $\nu$ that never visits $q$, but from which infinitely many accepting runs can separate that visit $q$ infinitely often. Let $\alpha_{i}$ denote an accepting run that separated from $\nu$ at time $i$.

Notice that whenever two different runs $\alpha_{i}$ and $\alpha_{j}$ with $i<j$ meet after $j$ in the same state, they can be continued in the same way. Consider now only such modified runs $\hat{\alpha}_{i}$, so we can assume that if $\hat{\alpha}_{i}$ and $\hat{\alpha}_{j}$ with $i<j$ are in different states at time $k>j$, they have not met yet after $j$. Let $S_{a}^{i}$ be the set of different $\hat{\alpha}_{j}$ with $j \leq i$ that are in state $a$ at time $i$.

Fix some time $i$ and pick $j>i$ such that at least $|Q|+1$ new runs $\hat{\alpha}_{k}$ with $i<k<j$ separated from $\nu$. Observe that either all new runs joined existing sets directly or at least two sets of runs previously occupying different states must have met and joined before $j$, i.e. $S_{a}^{i} \sqcup S_{b}^{i} \subseteq S_{c}^{j}$ for some states $a, b, c$ such that $a \neq b$. But then there is an infinite sequence $S_{a_{1}}^{1} \subseteq S_{a_{2}}^{2} \subseteq \ldots$ with $a_{i} \in Q$ that has a strict subsequence, as at most $|Q|$ different sets can exist at any time. Let $\hat{S}_{i}$ denote the set of such a sequence at time $i$. Each $\hat{\alpha}_{i}$ with $i \leq k$ that is in $\hat{S}_{k}$ hence also joins infinitely many $\hat{\alpha}_{j}$ with $j>i$ at some time $l>j$.

Pick some state $p$ visited by $\nu$ infinitely often and some run $\hat{\alpha}_{k}$ from $\hat{S}_{m}$ with $k \leq m$. Observe that $\hat{\alpha}_{k}$ has at least one state $r$ that it visits infinitely often at the same time as $\nu$ visits $p$. Pick a time $i>k$ when this happens, i.e. $\nu(i)=p$ and $\hat{\alpha}_{k}(i)=r$. Now pick a run $\hat{\alpha}_{l}$ from $\hat{S}_{m^{\prime}}$ such that $i<l<m^{\prime}$. Finally, pick a time $j>l$ such that $\hat{\alpha}_{k}$ and $\hat{\alpha}_{l}$ have joined, then visited $q$ together at least once and finally $\nu(j)=p$ while $\hat{\alpha}_{k}(j)=\hat{\alpha}_{l}(j)=r$.

Notice that all runs read the same finite substring $x$ of $w$ between $i$ and $j$. Further, $\nu$ witnesses a cycle $p \stackrel{x}{\rightarrow} p, \hat{\alpha}_{k}$ witnesses a cycle $r \stackrel{x}{\rightarrow} r$ on which $q \in F$ is visited and finally, as $\hat{\alpha}_{l}$ separates from $\nu$ after $i$ and joins $\hat{\alpha}_{k}$ before $j$, it witnesses a path $p \stackrel{x}{\rightarrow} r$. But this implies IDA $_{F}$ by Lemma 8 , violating the assumption.

Hence for each $q \in F$ there is a finite time after which all accepting runs that will visit $q$ infinitely often must have visited $q$ at least once. But then the number of accepting runs on $w$ must be finite. 


\section{A.2 Proof of PSPACE-completeness (Theorem 4)}

We will make use of the following two observations:

Lemma 9. If $\mathrm{da}\left(\mathcal{A}_{\mathrm{NBA}}, w\right)=k$, then there exists an ultimately periodic word $w^{\prime}=x y^{\omega} \in L\left(\mathcal{A}_{\mathrm{NBA}}\right)$ such that $\mathrm{da}\left(\mathcal{A}_{\mathrm{NBA}}, w^{\prime}\right) \geq k$.

Proof. Let $w \in L(\mathcal{A})$ with $\mathrm{da}(\mathcal{A}, w)=k$. Consider a sequence $t_{0}, t_{1}, \ldots$ of ordered tuples $t_{i} \in Q^{k}$ of states of all $k$ different accepting infinite runs at time $i$ and observe that as the number of different tuples is finite, there is an infinite sequence $i_{0}<i_{1}<\ldots$ such that all $t_{i_{j}}$ are equal. Now let $i_{a}$ be the first time in this sequence such that all $k$ runs already have separated and let $i_{b}>i_{a}$ be the first time after $i_{a}$ such that each run has visited an accepting state again. Then let $x$ be the prefix of $w$ that was read up to $i_{a}$ and $y$ the substring that was read between $i_{a}$ and $i_{b}$. By construction the ultimately periodic word $w^{\prime}=x y^{\omega}$ has at least as many accepting runs as $w$.

Lemma 10. If $\mathcal{A}$ is a finitely ambiguous NBA, then in each $S C C C, P(p, x, q)$ contains at most one path for every pair of states $p, q \in C$ and finite string $x$.

Proof. Assume there are $\pi_{1}, \pi_{2} \in P(p, x, q), \pi_{1} \neq \pi_{2}$. As we are in an SCC, there is a path $\pi_{q p} \in P(q, y, p)$. But then we have two different cycles $\hat{\pi}_{1}=\pi_{1} \pi_{q p}$ and $\hat{\pi_{2}}=\pi_{2} \pi_{q p}, \hat{\pi_{1}}, \hat{\pi_{2}} \in P(p, x y, p)$, which implies EDA.

Now we can adapt the algorithm presented in [7] to show PSPACE-completeness of this problem from NFA to NBA, proving the following result:

Theorem 4. Deciding whether $\operatorname{da}\left(\mathcal{A}_{\mathrm{NBA}}\right)>d$ for a given automaton $\mathcal{A}$ and $d \in \mathbb{N}$ is a PSPACE-complete problem.

Proof. First, we show that the problem can be decided in PSPACE.

Let $\mathcal{A}$ be a finitely ambiguous NBA with states $Q=\left\{q_{1}, \ldots, q_{n}\right\}$ and w.l.o.g. assume that $q_{1}$ is the initial state.

Define a set of matrices $\left\{T_{a}\right\}_{a \in \Sigma}$ with $T_{a}(i, j)=1$ if $q_{j} \in \Delta\left(q_{i}, a\right)$ and 0 otherwise. For each finite word $w \in \Sigma^{*}$, let $T_{w}=T_{w(1)} \cdot \ldots \cdot T_{w(n)}$. Then $T_{w}(i, j)$ is the number of different paths from $q_{i}$ to $q_{j}$ labelled with $w$ and hence $T_{w}(1, k)$ denotes the number of different runs that are in state $q_{k}$ after reading $w$.

Now let $C$ be an SCC of $\mathcal{A}$ and let $\pi_{C}(A)$ denote the restriction of matrix $A$ to the rows and columns that correspond to states in $C$. Then clearly the matrix $\pi_{C}\left(T_{w}\right)$ describes the number of different $w$-labelled paths between states of $C$. Remember that by Lemma 10 a finitely ambigious NBA has at most one path for each finite word between each pair of states that are within the same SCC, so $\pi_{C}\left(T_{w}\right)$ contains only 0 and 1 as values.

By Lemma 9 we can restrict ourselves to words of the form $x y^{\omega} \in L\left(\mathcal{A}_{\mathrm{NBA}}\right)$ and clearly we can choose $x y^{\omega}$ such that after reading $x$ all accepting runs are already separated and have reached their terminal SCC which is never left again. By choice of $x$ and Lemma 10 all accepting runs are continued unambiguously on $y^{\omega}$ after reading $x$. 
So to obtain the number of accepting runs, we need to sum up $T_{x}(1, k)$ for all states $q_{k}$ that lie on an accepting cycle when reading $y^{\omega}$. As there is only a finite number of such unambiguous continuation cycles, after a finite number of iterations of $y$ (least common multiple of all different cycle lengths and $|y|$ ) all runs are back in the state of the cycle where they started. So let $z=y^{j}$ for some $j \in \mathbb{N}$ such that $T_{z}(k, k)=1$ for every cycle from some $q_{k}$ back to $q_{k}$.

What is left to verify is that such a cycle is accepting. To do this, we define another set of matrices, $\left\{A_{a}\right\}_{a \in \Sigma}$ with $A_{a}(i, j)=2$ if $q_{j} \in \Delta\left(q_{i}, a\right), q_{j} \in F$, $A_{a}(i, j)=1$ if $q_{j} \in \Delta\left(q_{i}, a\right), q_{j} \notin F$ and 0 otherwise.

Now $A_{z}(i, i)>1$ iff the unique $z$-labelled cycle from $q_{i}$ to $q_{i}$ visits at least one accepting state. Using this, we obtain the following result: $\operatorname{da}(\mathcal{A})=\operatorname{da}\left(\mathcal{A}, x y^{\omega}\right)=$ $\Sigma_{i \in I} T_{x}(1, i)$ with $I=\left\{i \mid T_{z}(i, i)=1 \wedge A_{z}(i, i)>1\right\}$ for some choice of $x, y \in$ $\Sigma^{*}, j \in \mathbb{N}$ and $z=y^{j}$.

Hence, the following nondeterministic polynomial space algorithm decides whether $\operatorname{da}(\mathcal{A})>d$, by guessing a prefix $x$ that yields candidate paths and guessing $z$ to identify paths that are prefixes of an accepting run on the same word, using the reasoning above.

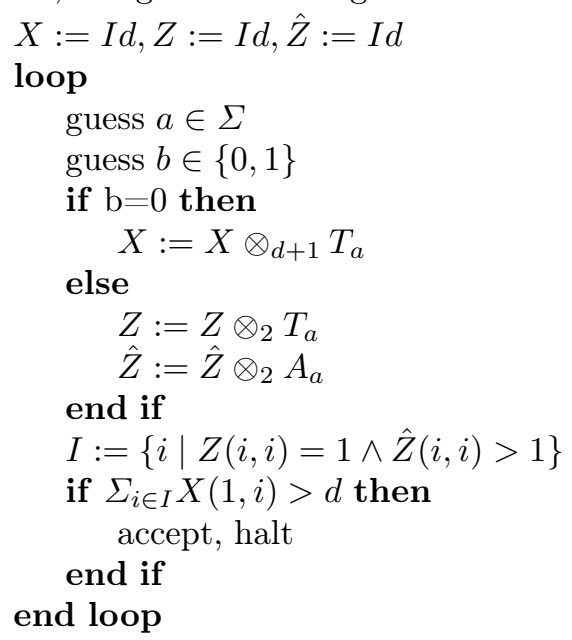

where $I d$ is the identity matrix and $\otimes_{n}$ is a matrix multiplication that identifies all integers $>n$ with $n$ (notice that this ensures the bounded space usage of the algorithm).

Now, we will show completeness. Deciding the ambiguity of a finitely ambiguous NFA is PSPACE-complete by [7]. We perform a reduction of the corresponding NFA problem to the NBA variant by introducing a fresh symbol \# and fresh state $q_{\#}$. The NBA $\mathcal{A}^{\prime}$ is defined by $\Sigma^{\prime}=\Sigma \cup\{\#\}, Q^{\prime}=Q \cup\left\{q_{\#}\right\}, Q_{0}^{\prime}=$ $Q_{0}, F^{\prime}=\left\{q_{\#}\right\}$ and $\Delta^{\prime}=\Delta \cup\left\{\left(q, \#, q_{\#}\right) \mid q=q_{\#} \vee q \in F\right\}$. It is easy to see that $L\left(\mathcal{A}_{\mathrm{NBA}}^{\prime}\right)=\left\{w \#^{\omega} \mid w \in L\left(\mathcal{A}_{\mathrm{NFA}}\right)\right\}$, i.e. each word of the NBA $\mathcal{A}^{\prime}$ is in one-to-one correspondence with a word of the NFA $\mathcal{A}$ that has the same number of accepting runs, as the only accepting SCC consists of $\left\{q_{\#}\right\}$, which is trivially unambiguous. Hence we decide $\operatorname{da}(\mathcal{A})>d$ by deciding $\operatorname{da}\left(\mathcal{A}^{\prime}\right)>d$ using the presented algorithm. 


\section{A.3 Proof of the lower bound (Theorem 6)}

Theorem 6. For each $n \in \mathbb{N}$ there exists an NBA with $n$ states such that each finitely ambiguous NBA accepting the same language has at least $2^{n}-1$ states.

Proof. In [13] this result is presented for the translation of NFA to polynomially ambiguous NFA and a corresponding family $\mathcal{A}_{n}$ of worst-case NFA of size $n$ is provided. Clearly translation to finitely ambiguous automata is not easier.

Pick some $n>0$. Let NBA $\hat{A}_{n}$ be defined by extending NFA $\mathcal{A}_{n}$ with a fresh symbol \# and additional transitions $\left\{\left(q_{F}, \#, q_{0}\right) \mid q_{F} \in F, q_{0} \in Q_{0}\right\}$. Let $\hat{\mathcal{B}}$ be a finitely ambiguous and trim NBA accepting the same language. We obtain an NFA $\mathcal{B}$ from $\hat{\mathcal{B}}$ by defining only the states with an outgoing \#-labelled transition as accepting and then removing those \#-labelled transitions. This NFA is also finitely ambiguous by Lemma 1 as removing transitions clearly can not increase ambiguity.

Let $w \in L\left(\mathcal{A}_{n}\right)$. Then by construction, at least the infinite word $(w \#)^{\omega}$ is in $L\left(\hat{\mathcal{A}}_{n}\right)$ and hence in $L(\hat{\mathcal{B}})$, so a \#-labelled transition must be possible after reading the prefix $w$ on some accepting run in $\hat{\mathcal{B}}$. By definition then we have $w \in L(\mathcal{B})$.

Let $w \notin L\left(\mathcal{A}_{n}\right)$. Then by construction there is no infinite word with prefix $w \#$ in $L\left(\hat{\mathcal{A}}_{n}\right)$ and hence neither in $L(\hat{\mathcal{B}})$. As $\hat{\mathcal{B}}$ is trim, a \#-labelled transition is not possible on any run after reading prefix $w$, and then by definition we have $w \notin L(\mathcal{B})$.

So we have $L(\mathcal{B})=L(\mathcal{A})$, which implies that $\mathcal{B}$ must have at least $2^{n}-1$ states by [13] and hence $\hat{\mathcal{B}}$ as well, by construction. 